\title{
AQUISIÇÃO FONOLÓGICA DE CRIANÇAS DE CLASSE SÓCIO ECONÔMICA ALTA
}

\section{Phonological acquisition in socio-economical high-class children}

\author{
Carla Ferrante ${ }^{(1)}$, John Van Borsel (2), Mônica Medeiros de Britto Pereira ${ }^{(3)}$
}

\begin{abstract}
RESUMO
Objetivo: investigar a aquisição fonológica em uma população de crianças com desenvolvimento normal. Métodos: foram avaliadas 240 crianças, de ambos os sexos, com idades entre três e oito anos. Foram realizadas análises relativas ao inventário fonético e percentual de consoantes corretas. Os dados foram analisados em relação à faixa etária e sexo. Resultados: aos três anos de idade os fonemas /p/, /b/, /t/, /d/, /k/, /g/, /m/, e /n/ já estão adquiridos e estabilizados no sistema fonológico das crianças. Os fonemas $/ \mathrm{f} /, / \mathrm{v} /, / \mathrm{s} /, / \mathrm{S} /, / \mathrm{z} /, / 3 /$, / $/ \mathrm{e} / \mathrm{n} / \mathrm{e}$ as africadas [t $\int$ ] e [d3]também são adquiridos nesta faixa etária apesar de ter sido encontrada uma grande variabilidade de produção entre as crianças. A aquisição do fonema / $/$ ocorre inicialmente na posição de onset simples (quatro anos) e posteriormente na posição de onset complexo (cinco anos), a aquisição do fonema / $/$ / em onset simples ocorre aos três anos e em onset complexo aos quatro anos e o fonema /R/ em onset simples é adquirido na faixa etária de três anos e na posição de coda aos quatro anos. Conclusão: desde a faixa etária de três anos, muitas crianças possuem o inventário fonético completo havendo, porém, uma grande variabilidade entre as crianças. Com relação ao PCC e ao PCC-R, concluiu-se que a média do percentual de consoantes corretas tem um crescimento significativo e gradual de acordo com o aumento da faixa etária. Em relação à variável sexo, não foi observada nenhuma diferença estatisticamente significante em nenhuma das análises realizadas nesta pesquisa.
\end{abstract}

DESCRITORES: Criança; Fala; Desenvolvimento da Linguagem

\section{INTRODUÇÃO}

O surgimento da linguagem expressiva ocorre durante os primeiros anos de vida da criança, período em que os fonemas são adquiridos e estabelecidos quanto às posições nas sílabas e nas palavras e de acordo com uma cronologia que é ao que parece similar para a maioria das crianças ${ }^{1}$. As consoantes plosivas e nasais são os primeiros segmentos consonantais a serem adquiridos pelas crianças com desenvolvimento fonológico normal, estando ambas adquiridas antes dos 2 anos de idade ${ }^{2,3}$, podendo $\mathrm{o} / \mathrm{n} / \mathrm{ser}$ adquirido um pouco mais tarde. Na língua inglesa foi observado ${ }^{4}$ que o inventário fonético de uma criança na faixa etária de três anos é composto

(1) Fonoaudióloga; Clínica Carla Ferrante; Mestre em Fonoaudiologia pela Universidade Veiga de Almeida.

(2) Fonoaudiólogo; Professor Titular da Gent Universiteit; Doutor em Neurolinguística pela Vrije Universiteit Brussel.

(3) Fonoaudióloga; Professora Adjunta da Universidade Veiga de Almeida; Doutora em Lingüística pela Universidade Federal de Minas Gerais. pelos fonemas /p/, /b/, /t/, /d/, /k/, /g/, /m/, /n/, /f/, /s/, ou seja, as consoantes plosivas, nasais e fricativas anteriores desvozeadas. Para outros autores ${ }^{5}$ todas as plosivas e as nasais já estão adquiridas na idade de três anos e seis meses.

As consoantes fricativas seguem as plosivas e as nasais na ordem de aquisição segmental das línguas naturais. Essa classe de sons caracteriza-se por conter tanto fonemas de aquisição inicial (/f/e $/ \mathrm{v} /$ ), como fonemas de aquisição mais tardia (/s/, $/ z /, / S /, / 3 /)^{2}$. As fricativas labiais são as primeiras a serem adquiridas na classe das fricativas ${ }^{2}$, assim como os fonemas sonoros são adquiridos antes do seus co-ocorrentes surdos ${ }^{3,6}$. As consoantes africadas aparecem no inventário fonético das crianças um pouco mais tarde aos $3: 6$ aos $4: 6^{3}$. No processo de aquisição fonológica da lingua inglesa foram observadas idades mais tardias para a aquisição destes sons ${ }^{4}$.

A classe das líquidas é a última a ser adquirida no Português e, dentro desse grupo de sons, as laterais são adquiridas antes das não-laterais. 
A primeira líquida lateral a se estabilizar na fala das crianças é o $/ 1 /$, a qual é dominada antes do surgimento da primeira líquida não-lateral /R/. O mesmo ocorre com os fonemas $/ K /$ e $/ \Omega /$, sendo o primeiro dominado antes do segundo ${ }^{2}$. A lateral alveolar/l/ é adquirida primeiro em posição de onset absoluto como em /' lata/, aos 2:8 e, alguns meses depois, é dominada em onset medial, como em / ' bala/, aos 3:0 ao passo que o $/ K /$ aparece aos 4 anos. As líquidas não laterais aparecem mais tardiamente no desenvolvimento fonológico ${ }^{7}$. A líquida /R/ está dominada aos 3:4 - 3:5, tanto em onset absoluto quanto em onset medial, seguida do / $/$ / na posição de onset simples aos 4:2. Em crianças com aquisição normal, as líquidas laterais são adquiridas antes das não-laterais e, dentro dessa classe, o / / / precede o $/ K /$, e o $/ R /$ geralmente aparece antes do $\mid \varsigma /{ }^{1}$. Observa-se na produção do $/ \varsigma /$, no entanto, grande variabilidade entre as crianças, estando este som realmente estabilizado aos 7 anos ${ }^{8}$.

São poucos os estudos que pesquisaram a diferença na aquisição fonológica de meninos e meninas. Em pesquisa ${ }^{9}$ realizada na Inglaterra com 684 crianças de 3 anos a 6 anos e 11 meses não foi observado diferença significativa nas faixas etárias mais baixas, porém, no grupo de crianças de faixa etária mais alta, as meninas apresentaram melhor precisão na produção dos sons.

A percepção da estrutura silábica segue uma ordem de aquisição ${ }^{10}$. A posição que o fonema $/ \varsigma /$ ocupa na sílaba é fator fundamental para a sua aquisição. O fonema $/ \varsigma /$ em onset simples é adquirido anteriormente ao $/ \mathrm{s} /$ em onset complexo, demonstrando a exigência de um maior grau de comple-

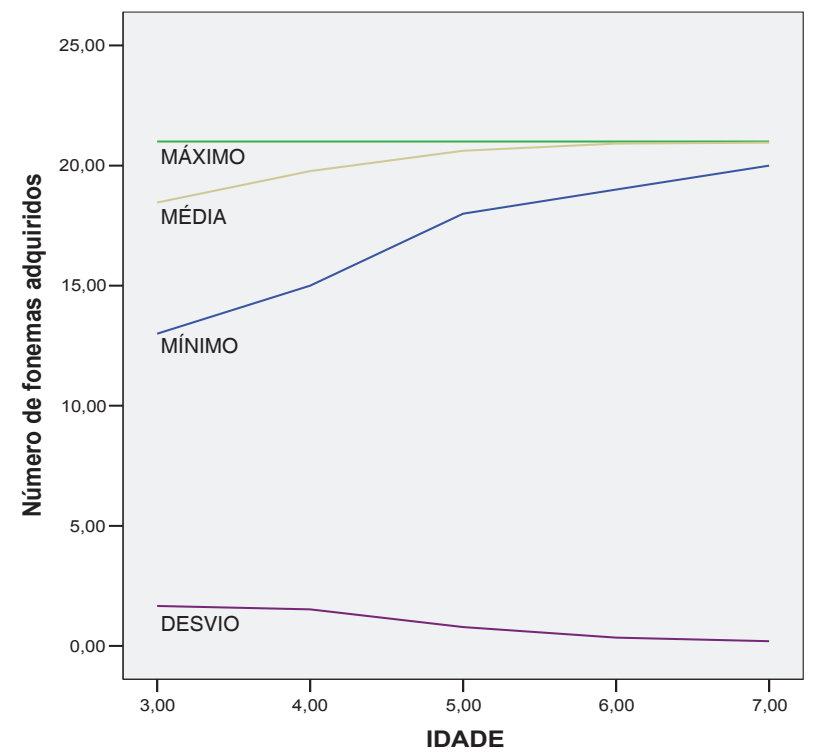

Figura 1 - Mínimo, máximo e média de fonemas adquiridos por faixa etária xidade para a produção do referido som neste contexto silábico ${ }^{8,10}$. Segundo alguns autores ${ }^{1}$ as estruturas silábicas são adquiridas na ordem $V$ e CV > CVC > CCV. Quanto à posição na sílaba e na palavra a ordem da aquisição é onset medial (início de sílaba dentro da palavra), seguido de coda final (final de sílaba final da palavra), onset inicial (início de sílaba início da palavra) e coda medial (final de sílaba dentro da palavra). Estudos apontam também para a importância do número de sílabas no processo de aquisição lingüística ${ }^{11}$.

O cálculo do PCC ${ }^{12}$ (percentual de consoantes corretas) é uma outra forma de avaliar o desenvolvimento fonológico. Além de ser um modo objetivo de determinar a gravidade de uma desordem, o PCC também pode proporcionar para os clínicos um critério quantitativo sobre a eficácia de um plano terapêutico ${ }^{13}$. Há também o PCC-R ${ }^{14}$ (PCC- revisado) que não considera as distorções como erros. Os índices de PCC ${ }^{5}$ observados em 40 crianças de três a $5: 6$ anos de idade, pareadas por sexo, sem queixa de distúrbios fonológicos foram: (3:0 a 3:6) = $79,77 \%$, (3:7 a 4:0) = 86,95\%, (4:1 a 4:6) $=86,67 \%$, $(4: 7$ a $5: 0)=91,16 \%$ e $(5: 1$ a $5: 6)=93,45 \%$. Outro estudo ${ }^{4}$ faz referência aos índices de PCC encontrados em crianças com desenvolvimento fonológico normal: 3 anos -3 anos 11 meses $=76,41 \%$, 4 anos -4 anos 11 meses $=82,45 \%, 5$ anos -5 anos 11 meses $=88,36 \%, 6$ anos -6 anos 11 meses $=$ $90,76 \%$ e 7 anos -7 anos 11 meses $=90,99 \%$. Ainda em outro estudo ${ }^{8}$ realizado com crianças de 3 a 8 anos analisou-se o PCC em relação ao

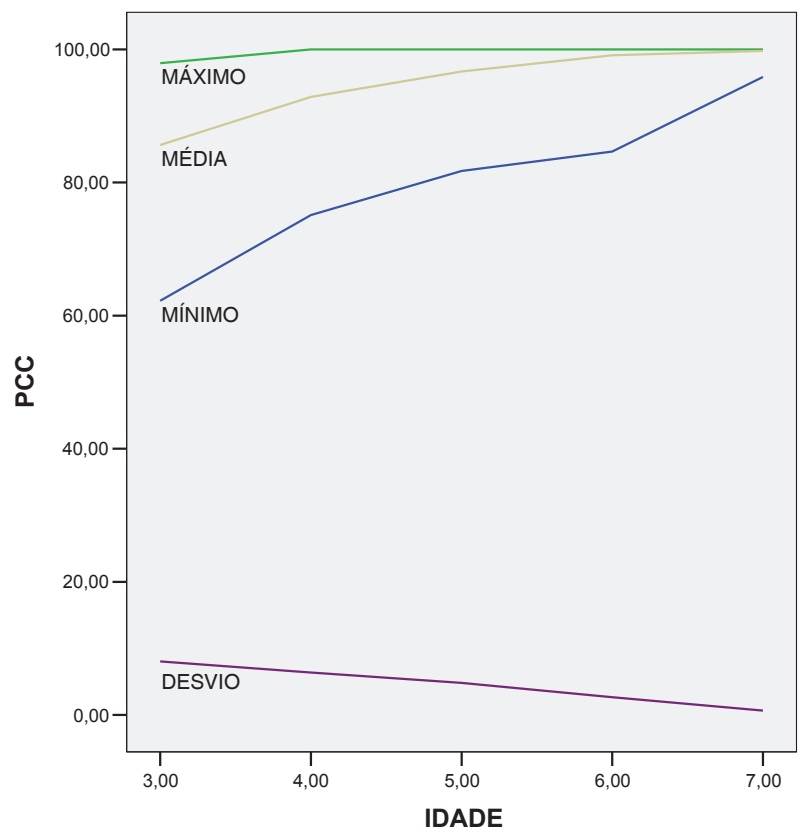

Figura 2 - Percentual de Consoantes Corretas por faixa etária 


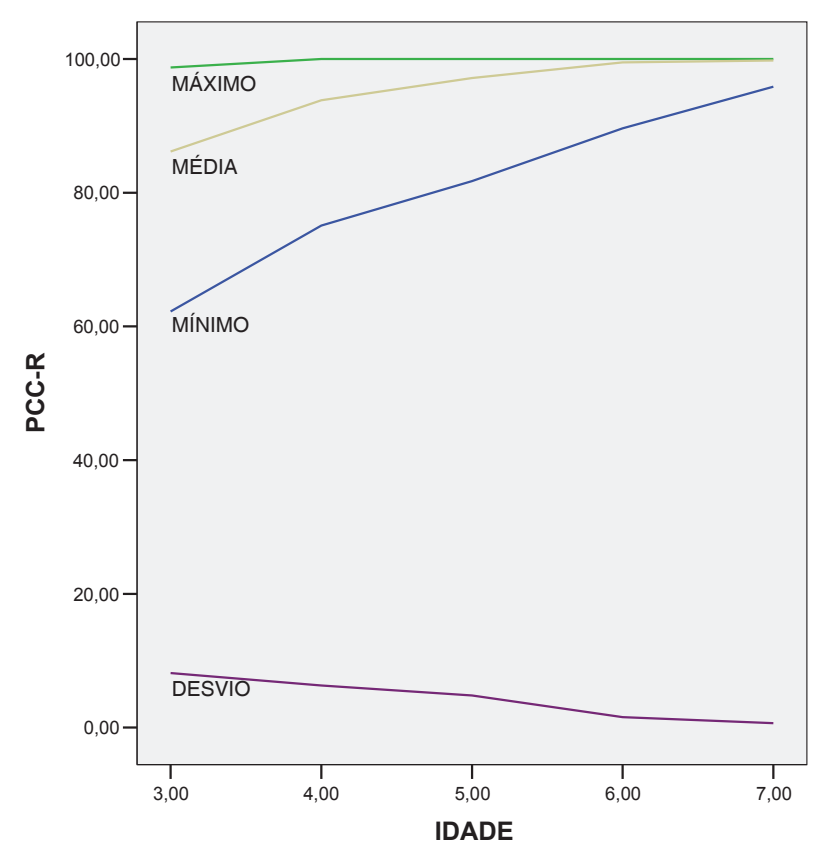

Figura 3 - Percentual de Consoantes Corretas Revisado por faixa etária

fonema $/ \mathrm{r} /$. As médias encontradas de acordo com as faixas etárias foram: 3 anos $=43,33 \% ; 4$ anos = 82,62\%; 5 anos: $73,12 \%$; 6 anos: $88,87 \%$ e aos 7 anos $=97,29 \%$. Outros autores ${ }^{15}$ realizaram uma pesquisa com 25 crianças com distúrbio fonológico com e sem história de transtorno de fala e linguagem no núcleo familiar e observaram que o grupo de crianças com história de problemas de fala e linguagem no núcleo familiar apresentou maior variabilidade nos índices de PCC-R. A média de PCC-R encontrada no grupo com história foi $69 \%$ e a média do grupo sem história familiar foi $72,50 \%$. Este estudo concluiu que os índices de PCC-R não diferenciaram o distúrbio fonológico em relação ao histórico familiar.

O desenvolvimento fonológico pode influenciar a plasticidade cerebral ${ }^{16}$ e o desenvolvimento lexical ${ }^{17}$. Estudos sugerem que crianças com atraso de fala de origem desconhecida podem ser portadores de dificuldades motoras na fala e também no processo de formulação lingüística ${ }^{18}$, outros estudos sugerem possiveis origens genéticas ${ }^{19}$. Tais dados reforçam ainda mais a importância de pesquisas nesta área. Neste sentido, o presente estudo tem por objetivo investigar o processo de aquisição fonológica em uma população de crianças de classe sócio econômica alta com desenvolvimento normal.

\section{MÉTODOS}

Foram avaliadas 240 crianças, de ambos os sexos, com idades entre três e oito anos, sendo a média de idade 5 anos e 6 meses. As crianças foram divididas em 05 grupos (3,0 a 3,11; 4,0 a 4,11; 5,0 a 5,$11 ; 6,0$ a 6,11 e 7,0 a 7,11 ) de forma que cada grupo fosse composto de 48 crianças, sendo 24 meninas e 24 meninos.

Os participantes eram estudantes de duas escolas particulares que aceitaram participar do estudo e que podem ser consideradas como representativas da classe média alta, do estado do Rio de Janeiro com base na localização na cidade e o preço da mensalidade escolar. Foram incluídas na pesquisa apenas as crianças que não apresentassem nenhum problema auditivo, neurológico ou de fala/linguagem, de acordo com a observação das professoras e coordenadoras pedagógicas e triagem realizada pelas pesquisadoras. As crianças que estavam em tratamento fonoaudiológico foram excluídas da amostra.

Para o desenvolvimento do instrumento de pesquisa, foram construídos inicialmente 06 diferentes corpora de palavras e aplicados em crianças de 4 a 10 anos, estudantes de escolas públicas do Rio de Janeiro e Minas Gerais, que não apresentassem nenhum problema relacionado à fala e/ ou à linguagem.

Visando investigar a melhor forma de apresentação das figuras, alguns exames utilizaram figuras retiradas de revistas e outros utilizaram desenhos feitos à mão. Após a aplicação destes exames, pode se observar que as figuras retiradas de revistas facilitavam a nomeação espontânea, pois são mais fidedignas à imagem real.

Para compor os diferentes corpora foram observados critérios relativos à estrutura sonora e à freqüência da palavra no vocabulário infantil. Muitas vezes uma palavra possui uma estrutura sonora que permite avaliar a produção de um ou mais fonema pela criança, mas não é uma palavra utilizada no vocabulário infantil, de forma que para que sua produção ocorra no momento da avaliação são necessárias muitas pistas. Na maioria dos casos as crianças não conseguiam acertar somente com as pistas fornecidas pelos examinadores, o que gerava a necessidade de imitação da palavra pronunciada pelo examinador. É o caso, por exemplo, da palavra "frutas", que possui uma estrutura sonora que permite avaliar a produção do grupo consonantal /fr/ não muito freqüente em nosso idioma. Ao olhar a figura das frutas, as crianças nomeavam as frutas isoladamente (banana, maçã, etc) e a palavra "frutas" não era produzida nem com o fornecimento das pistas. Este foi um dos motivos para a exclusão de palavras da avaliação, e devido a isso determinouse então que nenhum substantivo que designasse um grupo de outros substantivos faria parte do corpus final (animais, frutas, etc). 
Por fim foram selecionadas 79 palavras, respeitando os critérios de posição do som na palavra e na estrutura silábica (onset simples inicial, onset simples medial, onset complexo inicial, onset complexo medial, coda medial e coda final) e a freqüência no vocabulário infantil. $\mathrm{O}$ aspecto relativo à extensão da palavra também foi contemplado na confecção final do corpus.

As avaliações foram realizadas sempre por dois examinadores, em sala silenciosa e com o uso de gravadores analógicos para a análise dos resultados.

Com base nas observações feitas durante as coletas de dados realizadas com os diferentes corpora e posteriormente através de análise das gravações por duas examinadoras chegou-se ao corpus final de 79 palavras apresentadas impressas em papel fotográfico, tamanho 15X21. A opção pelas fotografias deu-se com o objetivo de facilitar o reconhecimento e a nomeação espontânea pela criança.

O protocolo de anotação dos dados foi composto por palavras e suas respectivas notações fonológicas. No referido protocolo o examinador deveria transcrever foneticamente a palavra pronunciada de maneira incorreta pela criança, respeitando as variações dialetais, marcando com um "i" no caso de imitação do modelo dado pelo examinador e anotando na coluna "trocas / omissões" o som omitido e/ou a substituição ocorrida. Neste mesmo protocolo encontram-se listados todos os processos fonológicos e para cada palavra, células em branco representam os processos fonológicos possíveis de ocorrência. Desta forma, o examinador deve marcar com um " $x$ " o processo fonológico que corresponda à produção feita pela criança.

A apresentação das palavras no protocolo foi feita por sorteio de forma que a organização ficasse aleatória, visando não dar pistas à criança quanto ao som inicial.

O protocolo de análise do PCC (Percentual de Consoantes Corretas) desta avaliação foi desenvolvido respeitando os procedimentos já utilizados em estudos anteriores ${ }^{12-14,20 .}$

As avaliações foram feitas pela $1^{\text {a }}$ pesquisadora, em sala silenciosa e com o auxílio de gravador digital (Mini Disc Sony). A notação fonética (normas do Alfabeto Fonético Internacional - IPA 1993) foi realizada durante a avaliação e reavaliada posteriormente utilizando a gravação digital em áudio. Os dados foram confrontados com a análise de um segundo avaliador com competência na área. Após a comparação, esclarecidas as divergências possíveis entre as duas notações, foi definida a notação final.
Com base na notação final foram iniciadas as análises relativas ao inventário fonético, onde foram definidos os fonemas que compõem o sistema de sons de cada criança. Foi organizado um quadro para cada criança com a porcentagem de ocorrência de cada som nas diferentes estruturas silábicas: onset simples, onset complexo e coda. O som foi considerado como adquirido quando sua ocorrência fosse igual ou superior a $75 \%$, padrão este estabelecido como suficiente para determinar a aquisição do fonema. Estes dados serviram de referência para as análises do total de fonemas adquiridos, respeitando a marca de $75 \%$, por faixa etária e sexo, e da produção correta de cada fonema nas diferentes estruturas silábicas por faixas etárias e sexo.

Em relação à análise do PCC, foi levantado o índice de consoantes corretas produzidas por cada criança. Os dados foram analisados posteriormente em relação à faixa etária e sexo. $\mathrm{Na}$ análise do PCC-R as distorções fonéticas não foram consideradas como erro. Também neste caso, foi realizada uma análise em relação à faixa etária e sexo.

Esta pesquisa foi aprovada pelo Comitê de Ética em Pesquisa da Universidade Veiga de Almeida, sob número de resolução 64/06 e todos os responsáveis pelos participantes assinaram um termo de consentimento livre e esclarecido concordando com as normas do estudo.

A análise estatística foi realizada no programa SPSS 13.0, utilizando-se os testes de KruskalWallis e Mann-Whitney.

\section{RESULTADOS}

Na Tabela 1 pode-se observar que o mínimo e a média de fonemas adquiridos aumentaram de acordo com a faixa etária; o máximo de fonemas adquiridos estabilizou-se desde a faixa etária de três anos, mantendo-se o teto de 21 fonemas (número total de fonemas consonantais do inventário fonético do Português Brasileiro - dialeto local) e o desvio padrão diminuiu gradativamente de acordo com o aumento da idade, o que demonstra que a variabilidade da produção dos fonemas entre as crianças diminui com o aumento da idade. Estas diferenças por faixa etária demonstraram ser significativas.

A Tabela 2 permite verificar que não houve diferença entre o mínimo, o máximo e a média de fonemas adquiridos por sexo.

A análise das produções corretas de cada fonema foi realizada em três etapas: primeiramente fez-se a análise do mínimo, do máximo e da média de produções corretas de cada som. Esta análise foi realizada com o total de crianças da pesquisa.

Através desta primeira análise, pode-se verificar que os fonemas /p/, /b/, /t/, /d/, /k/, /g/, /m/, e /n/ 
Tabela 1 - Mínimo, máximo e média de fonemas adquiridos $\mathrm{X}$ faixa etária

\begin{tabular}{lcccc}
\hline Idade & Mínimo & Máximo & Média & Desvio padrão \\
\hline 3 anos -3 anos 11meses & 13 & 21 & 18,46 & 1,66 \\
4 anos -4 anos 11meses & 15 & 21 & 19,77 & 1,52 \\
5 anos -5 anos 11meses & 18 & 21 & 20,62 & 0,79 \\
6 anos -6 anos 11meses & 19 & 21 & 20,92 & 0,35 \\
\hline 7 anos -7 anos 11meses & 20 & 21 & 20,96 & 0,21 \\
\hline
\end{tabular}

Chi-Square $=127,181 / \mathrm{dF}=4 / \mathrm{p}<0,001$

Tabela 2 - Mínimo, máximo e média de fonemas adquiridos $\mathrm{X}$ sexo

\begin{tabular}{lcccc}
\hline Sexo & Mínimo & Máximo & Média & Desvio padrão \\
\hline feminino & 13 & 21 & 20,26 & 1,45 \\
\hline masculino & 15 & 21 & 20,03 & 1,41 \\
\hline
\end{tabular}

Mann Whitney $Z=-1,815 / p=0,069$

apresentam um mínimo de produções corretas acima de $75 \%$, o que representa que na faixa etária de 3 anos estes sons já estão adquiridos. Os fonemas $/ \mathrm{f} /, / \mathrm{v} /, / \mathrm{s} /, / \mathrm{s} / \mathrm{e}$ os alofones [t $\left.\int\right]$, [d3] apesar de apresentarem um mínimo de produções que variam entre $0 \%$ e $39 \%$, possuem médias acima de $97,8 \%$ e desvios padrão abaixo de 10 , o que indica que o mínimo se refere a um número pequeno de crianças que tiveram uma produção baixa desses sons, não sendo este dado significativo para justificar a realização de uma análise mais detalhada.

Os fonemas /z/, /3/, /K/, /n/, / / /, /R/ e /l/ além de apresentarem um mínimo de produções em torno de 0 e médias abaixo dos demais sons, apresentaram desvios padrão acima de dez, o que indica que houve uma grande variabilidade no percentual de produção destes sons entre as crianças. Por este motivo foram realizadas duas análises mais detalhadas, sendo a primeira relativa ao mínimo, o máximo e a média de produções corretas destes sons, por faixa etária e a segunda relativa ao número de crianças que apresentaram produção desses sons acima de $75 \%$.

Como os fonemas / $/$ /, /R/ e /l/ encontram-se em diferentes posições nas estruturas silábicas, foi realizada a análise de acordo com cada posição.

Considerando a média de $75 \%$, pode-se observar que a aquisição do fonema / / / ocorre inicialmente na posição de onset simples (quatro anos) e posteriormente na posição de onset complexo (cinco anos). O mesmo ocorre com os fonemas / / / e /R/. A aquisição do fonema / / em onset simples ocorre aos três anos e em onset complexo aos quatro anos, o fonema /R/ em onset simples é adquirido na faixa etária de três anos e na posição de coda aos quatro anos.
Após a análise referente à faixa etária, foi realizada a comparação destes dados por sexo e não foi encontrada nenhuma diferença significativa.

Posteriormente foi realizada a análise referente ao total de crianças que apresentaram índices de produção dos referidos sons acima de $75 \%$ por faixa etária e sexo.

Ao verificar estas análises, pode-se observar que estes dados reforçam as idades de aquisição descritas anteriormente para os referidos sons nas diferentes posições.

Assim como nos itens anteriores, foram realizadas as análises em relação ao sexo e não foi encontrada nenhuma diferença estatisticamente significante.

Foi realizado também o levantamento do índice de consoantes corretas produzidas por cada criança (PCC e PCC-R). Tais dados foram comparados por faixa etária e sexo.

$\mathrm{Na}$ Tabela 3 pode-se observar que o mínimo e a média do PCC aumentaram de acordo com a faixa etária e o máximo alcançou o teto de $100 \%$ a partir da faixa etária de 4 anos. $O$ desvio padrão diminuiu de acordo com o aumento da idade das crianças, o que significa que a variabilidade entre as crianças da mesma faixa etária diminui com o aumento da idade. Estes dados são estatisticamente significantes

De acordo com estes resultados pode-se observar que não houve diferença estatisticamente significante em relação ao PCC entre os sexos.

$\mathrm{Na}$ Tabela 5 pode-se observar que o mínimo e a média do PCC-R aumentaram de acordo com a faixa etária, o máximo alcançou o teto de $100 \%$ a partir da faixa etária de quatro anos e o desvio padrão diminuiu com o aumento da idade das crianças. Estes dados são estatisticamente significantes. 
Tabela 3 - PCC X faixa etária

\begin{tabular}{lcccc}
\hline Idade & Mínimo & Máximo & Média & Desvio padrão \\
\hline 3 anos -3 anos 11meses & $62,24 \%$ & $97,92 \%$ & $85,65 \%$ & 8,04 \\
4 anos -4 anos 11meses & $75,10 \%$ & $100 \%$ & $92,85 \%$ & 6,37 \\
5 anos -5 anos 11meses & $81,74 \%$ & $100 \%$ & $96,69 \%$ & 4,82 \\
6 anos -6 anos 11meses & $84,65 \%$ & $100 \%$ & $99,12 \%$ & 2,68 \\
\hline 7 anos -7 anos 11meses & $95,85 \%$ & $100 \%$ & $99,78 \%$ & 0,66 \\
\hline
\end{tabular}

Chi-Square $=150,260 / \mathrm{dF}=4 / p<0,001$

Tabela 4 - Percentual de Consoantes Corretas X sexo

\begin{tabular}{lcccc}
\hline Sexo & Mínimo & Máximo & Média & Desvio padrão \\
\hline Feminino & $62,24 \%$ & $100 \%$ & $95,24 \%$ & 7,30 \\
\hline Masculino & $67,63 \%$ & $100 \%$ & $94,39 \%$ & 7,38 \\
\hline
\end{tabular}

Mann Whitney $Z=-0,689 / p=0,491$

Tabela 5 - Percentual de Consonates Corretas-Revisado X faixa etária

\begin{tabular}{lcccc}
\hline Idade & Mínimo & Máximo & Média & Desvio padrão \\
\hline 3 anos -3 anos 11meses & $62,24 \%$ & $98,75 \%$ & $86,16 \%$ & 8,15 \\
4 anos -4 anos 11meses & $75,10 \%$ & $100 \%$ & $93,85 \%$ & 6,31 \\
5 anos -5 anos 11meses & $81,74 \%$ & $100 \%$ & $97,17 \%$ & 4,80 \\
6 anos -6 anos 11meses & $89,63 \%$ & $100 \%$ & $99,50 \%$ & 1,56 \\
\hline 7 anos -7 anos 11meses & $95,85 \%$ & $100 \%$ & $99,78 \%$ & 0,66 \\
\hline
\end{tabular}

Chi-Square $=151,614 / \mathrm{dF}=4 / \mathrm{p}<0,001$

Tabela 6 - Percentual de Consoantes Corretas -Revisado X sexo

\begin{tabular}{lcccc}
\hline Sexo & Mínimo & Máximo & Média & Desvio padrão \\
\hline Feminino & $62,24 \%$ & $100 \%$ & $95,24 \%$ & 7,05 \\
\hline Masculino & $67,63 \%$ & $100 \%$ & $94,72 \%$ & 7,28 \\
\hline
\end{tabular}

Mann Whitney $Z=-1,109 / p=0,267$

A Tabela 6 representa a relação entre o PCC-R e o sexo. De acordo com estes resultados pode-se observar que não houve diferença entre o PCC-R e os sexos.

\section{DISCUSSÃO}

Os dados encontrados neste estudo evidenciaram que aos três anos de idade os fonemas $/ \mathrm{p} /, / \mathrm{b} /$, $/ \mathrm{t} /, / \mathrm{d} /, / \mathrm{k} /, / \mathrm{g} /, / \mathrm{m} /$, e /n/ já estão adquiridos e estabilizados no sistema fonológico das crianças. Ao verificar os dados referentes ao fonema $/ \mathrm{n} /$, observa-se que o mínimo de produção correta deste som estabilizou-se em $50 \%$ em todas as faixas etárias. Este dado pode ser justificado pela ocorrência de apenas duas palavras ("passarinho" e "caminhão") com o fonema $/ \mathrm{n} /$ na avaliação. Muitas crianças omiti- ram o referido fonema quando produziram a palavra "caminhão", provavelmente pelo fato desta palavra ter um som nasal antes do fonema $/ \mathrm{n} /$.

Os achados encontram respaldo em estudo ${ }^{2}$ que afirma que todas as plosivas e nasais estão adquiridas antes dos dois anos de idade, em outro estudo ${ }^{5}$ que observou que aos três anos e seis meses todas as plosivas e nasais estão adquiridas e na pesquisa de outro autor ${ }^{3}$ que observou que no estágio entre 1:6 e dois anos as crianças já adquiriram os fone$\mathrm{mas} / \mathrm{p} /, / \mathrm{b} /, / \mathrm{t} /, / \mathrm{d} /, / \mathrm{m} /$, e $/ \mathrm{n} /$ e aos dois anos e sies meses todas as plosivas e nasais estão adquiridas. Ainda outros autores ${ }^{4}$ relatam que aos três anos o inventário fonético das crianças é composto pelos fonemas /p/, /b/, /t/, /d/, /k/, /g/, /m/, /n/, /n/ /f/ e /s/ ou seja, consoantes plosivas, nasais e fricativas anteriores desvozeadas. 
Os resultados demonstraram que os fonemas $/ \mathrm{f} /, / \mathrm{v} /, / \mathrm{s} /, / \mathrm{S} / \mathrm{,} / \mathrm{z} / \mathrm{e} / 3 /$ e os alofones [t $\mathrm{S}]$ e [dz] também estão adquiridos nesta faixa etária apesar de ter sido encontrada uma grande variabilidade de produção entre as crianças. Dados semelhantes foram encontrados por outros pesquisadores ${ }^{2,6}$ que afirmam que as labiais são as primeiras a serem adquiridas na classe das fricativas, estando $\circ / \mathrm{v} /$ adquirido aos 1:8 e o /f/ aos 1:9, o fonema / / / aos 2:10 e o fonema /3/ aos 2:6. Em outro estudo ${ }^{2}$ foi observado que o fonema /s/ encontra-se adquirido aos 2:6 e o /z/ aos 2:0. Também segundo outro autor ${ }^{3}$ as fricativas /s/ e /f/ são adquiridas no estágio de 2:6 - 3:0 enquanto a fricativa / $/ \mathrm{S}$ e a africada [t $\int$ ] são adquiridas no estágio que vai dos $3: 0$ aos 3:6 e finalmente no estágio que vai dos 3:6 aos 4:6 as fricativas $/ z /, / 3 /$ e a africada [dz] aparecem no inventário fonético das crianças.

Estudos ${ }^{4}$ demonstraram, no entanto, dados diferentes, talvez por ter sido realizado com crianças falantes da língua inglesa. Neste estudo na faixa etária de 3 anos a 3 anos e 11 meses somente as fricativas /s/ e /f/ demonstraram estar adquiridas. Já as fricativas /f/, /v/, /s/, /z/ e / $/$ faziam parte do inventário fonético das crianças apenas na faixa etária de 4 anos a 4 anos e 11 meses. A fricativa /3/ demonstrou estar adquirida somente na faixa etária de 5 anos.

Em relação à aquisição das líquidas, observouse no presente estudo estudo que a aquisição do fonema / $/$ / ocorre inicialmente na posição de onset simples (4 anos) e posteriormente na posição de onset complexo (5 anos). O mesmo ocorre com o fonema $/ 1 /$, o qual aparece em onset simples aos 3 anos e em onset complexo aos 4 anos. Já o fonema /R/ em onset simples demonstra estar adquirido na faixa etária de 3 anos e na posição de coda aos 4 anos. Os dados também demonstram que o fonema I $\mathrm{K} /$ encontra-se adquirido aos 3 anos, apesar da grande variabilidade observada na produção deste som entre as crianças.

Dados semelhantes foram observados em um estudo ${ }^{2}$ concluindo que a classe das líquidas é a última a ser adquirida no Português e que dentro desse grupo de sons, as laterais são adquiridas antes das não-laterais. Em outro estudo ${ }^{7}$ foi observado que o /l/ é adquirido primeiro em posição de onset absoluto, aos 2:8 e aos 3:0 é dominado em onset medial. A líquida/R/ está dominada aos 3:4 3:5, tanto em onset absoluto quanto em onset medial, o fonema $/ K /$ é adquirido aos 4:0 e a líquida não-lateral $/ \varsigma /$ na posição de onset simples está adquirida aos 4:2. Os resultados do presente estudo também apontam para dados semelhantes, pois mesmo havendo encontrado a produção do / / / aos três anos, foi observado grande variabilidade entre as crianças desta faixa etária. Em outra pesquisa ${ }^{1}$ também foi observado que as líquidas laterais são adquiridas antes das não-laterais e, dentro dessas classes, o/l/precede o/K/, e o/R/ geralmente aparece antes do $/ \varsigma /$.

No que se refere ao $/ \varsigma /$, os resultados apresentam dados semelhantes à outras pesquisas ${ }^{8,15}$, em relação à posição na estrutura silábica e a idade de aquisição.

Com relação ao total de fonemas adquiridos, observou-se que desde a faixa etária de 3 anos, muitas crianças possuem o inventário fonético completo (com os 21 fonemas consonantais do inventário fonético do Português Brasileiro - dialeto local) havendo, porém, uma grande variabilidade entre as crianças, variabilidade esta, que diminui gradativamente de acordo com o aumento da faixa etária. Não foram encontrados dados similares na literatura para discussão deste aspecto.

Em relação ao PCC, foi observado que a média do percentual de consoantes corretas demonstra um crescimento significativo e gradual de acordo com o aumento da faixa etária. Esses dados não divergem muito dos resultados de outros autores ${ }^{5}$.

Ao comparar os dados do presente estudo com os dados de crianças falantes do inglês ${ }^{4}$ também com desenvolvimento fonológico normal, observouse índices maiores de PCC nas crianças brasileiras. Já em relação ao PCC-R, não foram encontrados estudos semelhantes para a comparação dos dados.

Em relação à variável sexo, não foi observada nenhuma diferença estatisticamente significante em nenhuma das análises realizadas nesta pesquisa.

Estes dados não estão em concordância com outros estudos ${ }^{9}$ que observaram que as meninas apresentaram melhor desempenho em algum momento do desenvolvimento fonológico.

\section{CONCLUSÃO}

Os dados permitem concluir que aos três anos de idade os fonemas /p/, /b/, /t/, /d/, /k/, /g/, /m/, e /n/ já estão adquiridos e estabilizados no sistema fonológico das crianças desta pesquisa. Os fonemas /f/, /v/, /s/, /S/, /t J/, /dz/, /z/, /z/ e / / / também já estão adquiridos nesta faixa etária apesar de ter sido encontrada uma grande variabilidade de produção entre as crianças pesquisadas. Em relação ao fonema $/ \mathrm{n} /$, apesar de terem sido encontrados índices baixos de produção média e índices de produção mínima estabilizados em $50 \%$ nas crianças, pode se considerar como um fonema adquirido aos três anos de idade.

A aquisição do fonema / $/$ / ocorreu inicialmente na posição de onset simples (quatro anos) e 
posteriormente na posição de onset complexo (cinco anos). O mesmo ocorreu com o fonema /1/. A aquisição do fonema / / / em onset simples ocorreu aos três anos e em onset complexo aos quatro anos. Já o fonema /R/ em onset simples foi adquirido na faixa etária de três anos e na posição de coda aos 4 anos.

Com relação ao total de fonemas adquiridos, pudemos concluir que desde a faixa etária de três anos, muitas crianças já possuíam o inventário fonético completo (com os 21 fonemas consonantais do inventário fonético do Português Brasileiro
- dialeto local) tendo sido observada porém, uma grande variabilidade entre as crianças, variabilidade esta, que diminuiu gradativamente de acordo com o aumento da faixa etária.

Com relação ao PCC e ao PCC-R, os dados encontrados nesta pesquisa evidenciaram que a média do percentual de consoantes corretas teve um crescimento significativo e gradual de acordo com o aumento da faixa etária.

Em relação à variável sexo, não foi observada nenhuma diferença estatisticamente significante em nenhuma das análises realizadas nesta pesquisa.

\section{ABSTRACT}

Purpose: to investigate phonological acquisition in population of children with normal development. Methods: the participants of this study were 240 children of both genders, between 3 and 8-year old. Data were analyzed with respect to age and gender. Results: the results allow to conclude that at the age of 3 the phonemes $/ \mathrm{p} /, / \mathrm{b} /, / \mathrm{t} /, / \mathrm{d} /, / \mathrm{k} /, / \mathrm{g} /, / \mathrm{m} /$, and $/ \mathrm{n} /$ are already acquired and stabilized in the phonological system of the children. Also the phonemes $/ \mathrm{f} /, / \mathrm{v} /, / \mathrm{s} /, / \mathrm{S} /, / \mathrm{z} /, / 3 /, / \mathrm{K} /$ and $/ \mathrm{n} /$ and the affricates $\left[t \int\right]$ e $[\mathrm{d} 3]$ are already acquired at that age although we found a great production variability between children. The acquisition of the phoneme $/ \Omega /$ occurs initially in simple onset (age 4) and afterwards in complex onset (age 5), the acquisition of the phoneme /1/ in simple onset occurs at age 3 and in complex onset at age 4 and the phoneme /R/ in simple onset is acquired at age of 3 and in final position at the age of 4 . Conclusion: as for the total number of phonemes acquired, it can be concluded that from the age of 3 many children have a complete phonetic inventory, there is a great variability among children. As far as PCC and PCC-R is concerned, the mean percentage of correct consonants increases significantly and gradually while age increases. With regard to gender, not a single significant difference was found for any of the analyses performed in the current study.

KEYWORDS: Child; Speech; Language Development

\section{REFERÊNCIAS}

1. Keske-Soares M, Blanco APF, Mota HB. O desvio fonológico caracterizado por índices de substituição e omissão. Rev Soc Bras Fonoaudiol. 2004; 9(1):10-8.

2. Lamprecht RR. Aquisição fonológica do Português: perfil de desenvolvimento e subsídios para terapia. Porto Alegre: Artmed; 2004. 232p.

3. Grunwell P. Assessment of phonology. In: Grundy $\mathrm{K}$. Linguistics in clinical practice. 2. ed. London: Whurr Publishers; 1995.p.108-36.

4. McLeod S, Bleile K. Neurological and developmental foundations of speech acquisition. Am Speech Lang Hear Assoc Convent. Chicago; nov 2003.

5. Wertzner HF, Dias TA. PCC de crianças sem queixa de distúrbios de comunicação. Anais do VIII Congresso Brasileiro de Fonoaudiologia Recife PE; Out 2000.
6. Oliveira CC. Perfil da aquisição das fricativas /f/, $/ \mathrm{v} /, \mid \check{S} /$, /Žl do Português brasileiro: um estudo quantitativo. Porto Alegre: Letras de Hoje; 2003. p. 97-110.

7. Hernandorena CLM, Lamprecht RR. A aquisição das consoantes líquidas do Português. Porto Alegre: Letras de Hoje; 1997. p.7-22.

8. Teixeira AVFAL. Aquisição da líquida nãolateral /R/ em crianças de 3:0 a 7:11 anos em uma escola de classe média do município de Niterói. [dissertação]. Rio de Janeiro (RJ): Universidade Veiga de Almeida; 2006.

9. Dodd B, Holm A, Hua Z, Crosbie S. Phonological development: a normative study of British Englishspeaking children. Clin Linguist Phon. 2003; 17(8):617-43.

10. Miranda AR. A aquisição do 'r': uma contribuição à discussão sobre seu status fonológico. [monografia] Porto Alegre (RS): Pontifícia Universidade Católica do Rio Grande do Sul; 1996. 
11. Vigário M, Freitas MJ, Frota S. Grammar and frequency effects in the acquisition of prosodic words in European Portuguese. Lang Speech. 2006; 49(Pt2):175-203.

12. Shriberg LD, Kwiatkowski J. Phonological disorders I: a diagnostic classification system. J Speech Hear Disord. 1982 aug; 47(3):256-41.

13. Pena-Brooks A, Hedge MN. Assessment and treatment of articulation and phonological disorders in children: a dual-level text. Texas: Pro-ed an International Publisher; 2000.

14. Shriberg LD, Austin D, Lewis BA, McSweeny JL, Wilson DL. The percentage of consonants correct (PCC) metric: extensions and reliability data. J Speech Lang Hear Res. 1997 aug; 40(4):708-22.

15. Papp ACCS, Wertzner HF. O aspecto familial e o transtorno fonológico. Pró-Fono. 2006; 18(2):151-60.
16. Werker JF, Tees RC. Speech perception as a window for understanding plasticity and commitment in language systems of the brain. Dev Psychobiol. 2005; 46(3):233-51.

17. Pierrehumbert JB. Phonetic diversity, statistical learning, and acquisition of phonology. Lang Speech. 2003; 46(Pt2-3):115-54.

18. Flipsen Junior $P$. Articulation rate and speechsound normalization failure. J Speech Lang Hear Res. 2003 june; 46(3):724-37.

19. Lewis BA, Shriberg LD, Freebairn LA, Hansen AJ, Stein CM, Taylor HG, lyengar SK. The genetic bases of speech sound disorders: evidence from spoken and written language. J Speech Lang Hear Res. 2006 dec; 49(6):1294-312 .

20. Wertzner HF, Amaro L, Teramoto SS. Gravidade do distúrbio fonológico: julgamento perceptivo e porcentagem de consoantes corretas. Pró-Fono. 2005; 17(2):185-94.

RECEBIDO EM: 25/02/2008

ACEITO EM: 05/06/2008

Endereço para correspondência:

Rua Ipanema 197, ap. 1301

Rio de Janeiro - RJ

CEP: 22631-390

Tel: (21) 3325-9660

Fax: (21) 3325-9660

E-mail: caferrante@ rjnet.com.br 